\title{
Adjusted permutation method for multiple attribute decision making with meta-heuristic solution approaches
}

\author{
Hossein Karimi ${ }^{a}$, Alireza Rezaeinia ${ }^{b}$ \\ ${ }^{a}$ Department of Industrial Engineering, Shahed University, Tehran, Iran \\ ${ }^{b}$ Department of Industrial Engineering, Tehran University of Teacher Training, Tehran, Iran

\begin{tabular}{|c|c|}
\hline ART I C LE I NFO & A B S T R A C T \\
\hline $\begin{array}{l}\text { Article history: } \\
\text { Received } 1 \text { July } 2010 \\
\text { Received in revised form } \\
13 \text { October } 2010 \\
\text { Accepted } 14 \text { October } 2010 \\
\text { Available online } 14 \text { October } 2010 \\
\text { Keywords: } \\
\text { MADM } \\
\text { Adjusted permutation } \\
\text { Tabu search }\end{array}$ & $\begin{array}{l}\text { The permutation method of multiple attribute decision making has two significant deficiencies: } \\
\text { high computational time and wrong priority output in some problem instances. In this paper, a } \\
\text { novel permutation method called adjusted permutation method (APM) is proposed to } \\
\text { compensate deficiencies of conventional permutation method. We propose Tabu search (TS) } \\
\text { and particle swarm optimization (PSO) to find suitable solutions at a reasonable computational } \\
\text { time for large problem instances. The proposed method is examined using some numerical } \\
\text { examples to evaluate the performance of the proposed method. The preliminary results show } \\
\text { that both approaches provide competent solutions in relatively reasonable amounts of time } \\
\text { while TS performs better to solve APM. }\end{array}$ \\
\hline
\end{tabular}

(C) 2011 Growing Science Ltd. All rights reserved.

\section{Introduction}

There are many human-made decisions which are influenced by various conflicting factors. Organizations are typical examples of environments where different segments (departments) are in consistent conflict in terms of organizational objectives. In such a conflicting environment, multiple criteria decision making (MCDM) techniques are basically developed to select or rank decision alternatives. The existing conflict among goals of a decision maker implies that full attainment to a particular goal may jeopardize reaching the other objectives. In the past, business and industry decisions were used to have merely two determinants: the "boss", and the resulting profit. Today, however, decision making may involve many people with multiple and conflicting criteria. To cope with this complexity, researchers are consistently looking for more capable MCDM techniques which could better capture the real situation of a decision making process.

In MCDM, there are two types of criteria: objectives and attributes. Based on the type of criteria, MCDM problems can be classified into two categories: Multiple objective decisions making (MODM) and multiple attribute decision making (MADM). The basic difference between MODM and MADM problems is that the former concentrates on a continuous decision space while the latter

* Corresponding author. Tel.: +0989112713453

E-mail addresses: , Email:hkarimi@shahed.ac.ir (H. Karimi)

(c) 2011 Growing Science Ltd. All rights reserved. doi: 10.5267/j.ijiec.2010.07.002 
focuses on problems with discrete decision spaces (Yoon \& Hwang, 1981). MADM methods are classified into the following groups: 1) Compensatory methods where a production with high expenditure but good quality is compensated because of its high quality (Yoon \& Hwang, 1981). ELECTRE, MDS, MRS, TOPSIS, SAW, Linear assignment are examples of this method. 2) Non compensatory methods where the attributes are separated. For instance, for taking driving license non compensative important factors such as normal eye test, driving rule test and practical driving examination are used where one's strength in one of the tests does not compensate the others. Dominance, Lexicography, Elimination, Permutation are examples of this kind of model (Korhonen et al., 1992).

Each MADM methodology has its own limitations and attributes, and the decision maker cannot use one model for all decision-making problems. Using MADM methodology to prioritize various alternatives of a decision problem requires considering both the characteristics of the preferred methodology and the attributes of the problem itself. Nevertheless, there is always a chance of reaching to wrong priorities of the alternatives. The technique for order preference by similarity to ideal solution (TOPSIS) is a classical method to solve multi-criteria decision-making problem which was first developed by Hwang and Yoon (1981) and subsequently discussed by many such as Chu (2002), Olson (2004) and Peng (2000). According to TOPSIS, alternatives are chosen based on the shortest distance from the positive ideal solution (PIS) and the farthest distance from the negative ideal solution (NIS). The PIS has the best measures over all attributes, while the NIS has the worst measures over all attributes. TOPSIS was originally presented in the context of multiple criteria decision making, where the relative importance decision maker preference was a factor and subjective weights were inputs.

The analytical hierarchy process (AHP) method, as another MADM method, developed by Saaty (1990), is based on three principles: decomposition, comparative judgment and synthesis of priorities. Based on the decomposition principle, the decision problem is decomposed into a hierarchy that captures the necessary elements of the problem. The ELECTRE I method, originally introduced by Roy (1968), was built for multi-criteria choice problem where the primary aim is to obtain ranking for various alternatives. ELECTRE II was developed by Roy and Bertier (1971) where it represented an improvement on ELECTRE I. Just a few years later, ELECTRE III for ranking actions was devised by Roy (1978). Another ELECTRE method, known as ELECTRE IV, was introduced by Hugonnard and Roy (1982) as a technique for ranking the alternatives in a real-world project. The new method was equipped with an inserted outranking relationships framework. ELECTRE III is used when it is possible and desirable to express the quantity of the relative importance of criteria and ELECTRE IV is applied when this quantification is not possible.

Permutation method (PM) is one of the MADM techniques to find the best linear ordering of the alternatives. It is often difficult to reach the final result by PM since the method needs to spend tremendous amount of CPU-time which increases exponentially as the number of the alternatives increases. Also, Rinnooy (1976) proved that if the number of alternatives increases, the problem becomes NP-hard. Among all MADM approaches, permutation method is a technique developed to rank decision alternatives based on decision matrix and weights of criteria. This method is applied on a variety of industrial problems including location and allocation of factories in national or international level, establishment of efficient production and non-production units in factory environment, suitable layout design for equipment and machinery production units, selection of suppliers, etc. There are different goals and criteria which could be considered in the decision-making process such as working conditions, production requirements, practical constraints, and personnel requirements.

As an early application, the permutation method was used by Paelinck (1977) in an airport location problem. Ancot and Paelinck (1982) applied permutation method in urban resource management related to water supply and garbage grave, public transport, and public services. Blair and Karnisky 
(1994) applied the method for analysis of recorded electrical wave datasets of brain. Chin and Haughton (1996) proposed permutation test for analyzing the student learning evaluations. Permutation methods were also applied by Pantazis et al. (2003) for the analysis of MEG data in reconstructed cortical maps of brain activation. Turskis (2008) used this method for contractor's evaluation. Chen and Wang (2009) presented interval-valued fuzzy permutation (IVFP) methods for solving MADM problems based on interval-valued fuzzy sets and showed how this method could be used in group decision makings. For more reviewing MCDM literature, interested readers can study Figueira et al. (2005).

In this paper, an adjusted permutation method (APM) is proposed to correct shortcomings of the classical permutation method. Since APM has the same complexity degree as the classical permutation method, tabu search (TS) and particle swarm optimization (PSO) techniques are proposed to handle large instances of the problem.

The remainder of this paper is organized as follows. Section 2 is dedicated to an introduction to the classical permutation method. The APM is proposed in Section 3. Two meta-heuristic techniques including TS and PSO are described in Section 4 to handle APM for large instances of the problem. Numerical examples are presented in Section 5. Finally, the paper is concluded in Section 6.

\section{Classical permutation method}

The permutation method for MADM problem was first proposed by Jacquet-Lagreze (1969). It is based on the permutation of decision alternatives: If $m$ alternatives are available, then $m$ ! permutations can be generated. This method calculates a rate for each permutation and eventually chooses the permutation with the highest rate as the preferable permutation. Suppose there are $m$ alternatives $\left(A_{1}, A_{2}, \ldots, A_{m}\right)$ and the problem has $n$ criteria $\left(X_{1}, X_{2}, \ldots, X_{n}\right)$. Then the decision matrix (D) can be formed with alternatives and criteria in rows and columns, respectively. In general, the importance of each criterion is different.

For instance suppose that there are three alternatives $(m=3)$ which are displayed with $A_{1}, A_{2}$ and $A_{3}$. In this case, there are $3 !=6$ permutations as $P_{1}=\left\{A_{1}, A_{2}, A_{3}\right\}, P_{2}=\left\{A_{1}, A_{3}, A_{2}\right\}, P_{3}=\left\{A_{2}, A_{1}, A_{3}\right\}$, $P_{4}=\left\{A_{2}, A_{3}, A_{1}\right\}, P_{5}=\left\{A_{3}, A_{1}, A_{2}\right\}$, and $P_{6}=\left\{A_{3}, A_{2}, A_{1}\right\}$.

In order to choose the best permutation, we first define the concordance $\left(C_{k l}\right)$ and the discordance $\left(D_{k l}\right)$ sets. These sets are generated based on pairwise comparison of alternatives $k$ and $l$ regarding the criteria. $a_{k j}$ is defined as the performance of $k$ th alternative in $j$ th criterion in the decision matrix. Suppose criterion $j$ has positive effect on our decision. Now if $a_{k j} \geq a_{l j}$, Then $j$ will be in $C_{k l}$ set, otherwise $j$ will be in $D_{k l}$ set. Mathematically speaking, the concordance and discordance sets are defined as:

$$
\begin{aligned}
& C_{k l}=\left\{j \mid a_{k j} \geq a_{l j}\right\} \quad k, l=1,2, \ldots, m \quad k \neq l \\
& D_{k l}=\left\{j \mid a_{k j} \leq a_{l j}\right\} \quad k, l=1,2, \ldots, m \quad k \neq l
\end{aligned}
$$

After generating $C_{k l}$ and $D_{l k}$ for a permutation, the rate of that permutation is calculated as follows,

$$
R_{i}=\sum_{j \in C_{k l}} w_{j}-\sum_{j \in D_{k l}} w_{j}, \quad i=1, \ldots, m !
$$


where $R_{i}$ and $w_{j}$ denote the rate of permutation $P_{i}$ and the weight of criterion $j$, respectively. After calculating these rates for all permutations, the permutation with the highest rate is selected as the best priority sequence of decision alternatives.

In the pairwise comparison of alternatives, the classical permutation method can only consider the priority of an alternative over the other one, and not the degree by which this priority exists. This shortcoming can be addressed through the following example. Suppose there are three alternatives and three criteria in a decision matrix given by Table 1 .

Table 1

A sample decision matrix

\begin{tabular}{lccc}
\hline & \multicolumn{3}{c}{ Criterion } \\
\cline { 2 - 4 } Alternative & $1(-)$ & $2(+)$ & $3(+)$ \\
\hline 1 & 200 & 5 & 2400 \\
2 & 300 & 5 & 2420 \\
3 & 350 & 3 & 2000 \\
\hline
\end{tabular}

The first criterion has a negative effect and the other two criteria have positive effects on the decision. The weights of criteria are given as $0.3,0.4$, and 0.3 , respectively. Assuming a linear utility function, it is clear that the alternative 1 is better than alternative 2. The classical permutation method, however, cannot underscore this dominance. It calculates the same rate of 2 for permutations $\left\{A_{1}, A_{2}, A_{3}\right\}$ and $\left\{A_{2}, A_{1}, A_{3}\right\}$ whereas the former is clearly the best permutation of this problem. To address this drawback, the APM is proposed in the next section.

\section{Adjusted Permutation Method}

In APM, the rate of each permutation is calculated as follows,

$R_{i}^{\prime}=\sum_{j \in C_{k l}} w_{j}\left(\frac{\left|a_{k j}-a_{l j}\right|}{a_{j}^{\max }-a_{j}^{\min }}\right)-\sum_{j \in D_{k l}} w_{j}\left(\frac{\left|a_{k j}-a_{l j}\right|}{a_{j}^{\max }-a_{j}^{\min }}\right), \quad i=1, \ldots, m !$

where $a_{j}^{\max }$ and $a_{j}^{\min }$ are the maximum and the minimum values of the $j$ th criterion, respectively. The first and the second terms on the right hand side of (4) calculate the sum of weighted standard proportional priority of each permutation using concordance and discordance sets, respectively. Using the previous example, the adjusted rate of permutation $\left\{A_{1}, A_{2}, A_{3}\right\}$ is calculated as 1.9714 versus 1.6 obtained for permutation $\left\{A_{2}, A_{1}, A_{3}\right\}$. The exact algorithm used by APM to solve MADM problems has the same complexity as the classical permutation method. The computational time grows exponentially as the number of alternatives increases. Therefore, meta-heuristic procedures are required to handle large instances of the problem.

\section{Meta-heuristic procedures for APM}

In their original definition, meta-heuristics are solution methods that coordinate an interaction between higher level strategies and local improvement procedures to make a useful process of escaping from local optima and to achieve a robust exploration of solution space. These methods may also embed procedures that utilize strategies for overcoming the trap of local optimality in complex solution spaces, especially those procedures that employ one or more neighborhood structures as a tool of defining acceptable moves to transition from one solution to another, or to build or destroy solutions.

In this paper, two meta-heuristics are suggested to solve APM in reasonable amount of computational resources. The first is based on TS and the second is based on PSO. To the best of our knowledge, 
these meta-heuristics have never been applied to the permutation method in MADM area. But, these are used in other problems like sequencing problem. There are different works such as Nowicki and Smutnicki (1996) and Grabowski and Wodecki (2004) to apply TS for accelerating the process of permutation method in sequencing problem. Grabowski and Pempera (2007) used TS algorithm to develop a method for minimizing makespan in a flowshop problem. Liao and Huang (2010) proposed a method to solve a sequencing problem that simultaneously uses two TS algorithms with adaptation of permutation method. Tasgetiren et al. (2007) presented PSO to solve the permutation flowshop sequencing problem with the objectives of minimizing makespan and the total flowtime of jobs. This could motivate us to use TS and PSO algorithm for solving permutation method in other areas.

\subsection{TS for APM}

There are many important applications in different fields of sciences where it is practically impossible to detect the optimal solution. In such cases, one of the relevant choices is to use meta-heuristic approaches such as TS or PSO. TS was first proposed by Glover $(1989,1990)$ and it has been dramatically changing the ability of solving problems of practical significance. The pseudo code of the proposed TS for APM is as follows:

1. Insert decision matrix and weights, Calculate the minimum and maximum values of rating of each alternative with respect to each criterion

2. Generate a random permutation and calculate its rate $R$ from (4), Entitle this permutation and its rate as Best_Per and Best_R, respectively

3. Set Max_Iter, Max_STM (Short Term Memory) and Iter=1, Insert LTM (Long Term Memory) as a matrix with all elements equal to 1

4. Generate Random matrix using (5):

$\operatorname{Random}(i, j)=\frac{\operatorname{rand}(0,1)}{\operatorname{LTM}(i, j)}$

5. Set N1 and N2 as the row and the column of the maximum value in the Random matrix, respectively, if their exchange is in the STM go to step 4

6. Exchange N1 with N2 and set as Per and calculate the rate of this new permutation, Insert this exchange in STM and eliminate the last exchange in STM like (M1, M2)

7. $\operatorname{LTM}(M 1, M 2)=\operatorname{LTM}(M 1, M 2)+1$

8. If $R>$ Best_R, then Best_R=R and Best_Per=Per

9. Iter $=$ Iter +1. If Iter $>$ Max_Iter, then the final solution is Best_R and Best_per; else go to step 4

There are two parameters in proposed TS. Max_Iter and Max_STM must be tuned before executing the procedure. The important factor in tuning these parameters is the number of alternatives (NOA). The tuning empirical formulas are expressed in consequence of some experiments by considering the NOA. Hence, the suitable performance of proposed TS can be achieved by setting these parameters as: $M a x_{-}$Iter $=40 \times N O A, M a x_{-} S T M=\lfloor N O A \div 5\rfloor$. Randomization and diversification are considered using Eq. (5) by choosing the row and the column with the maximum value in the Random matrix, respectively. The procedure is depicted in Fig. 1. 

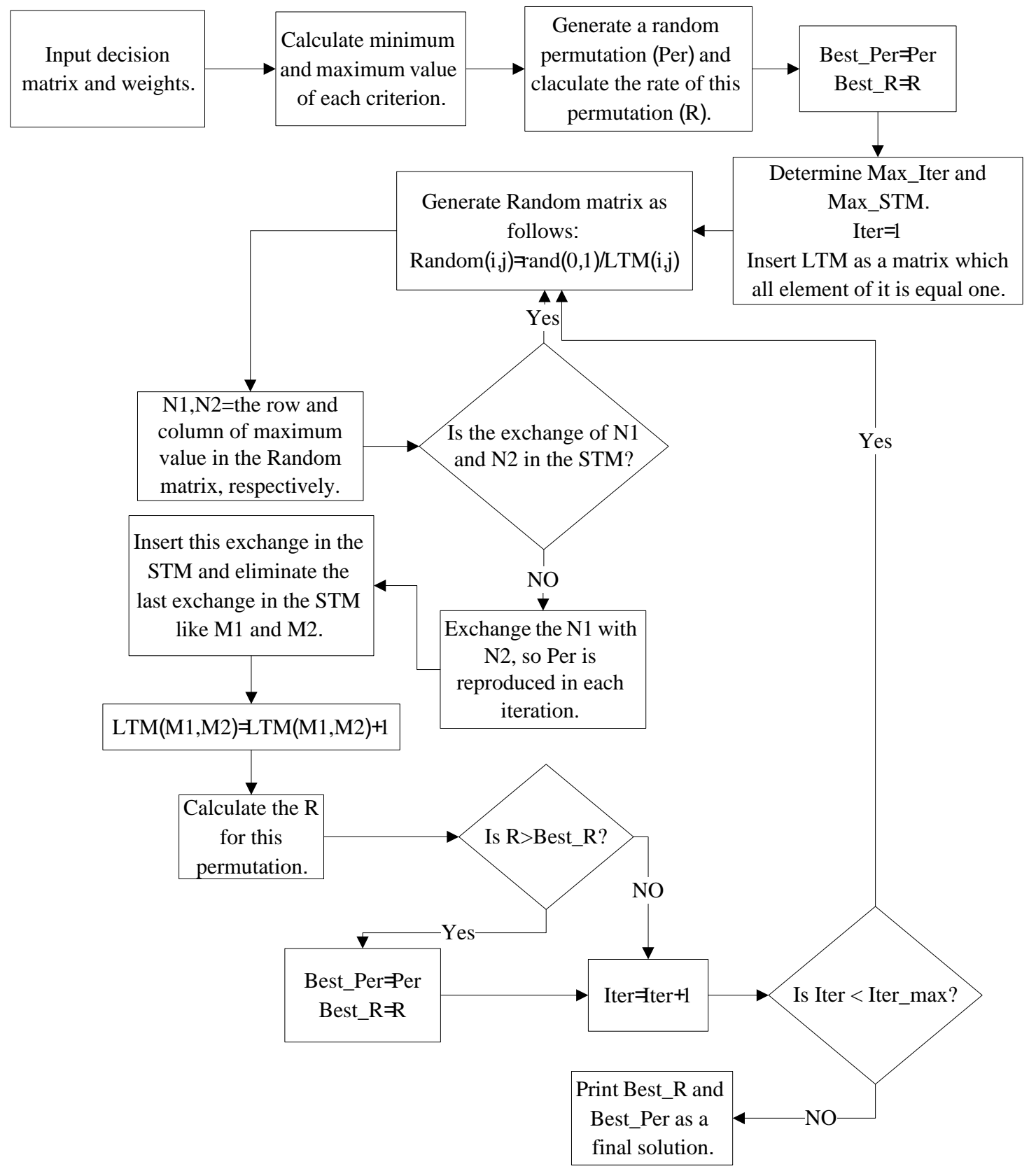

Fig. 1. Flowchart of the proposed TS

\section{2. $P S O$ for $A P M$}

PSO is a significant member of swarm intelligence. It was proposed by Kennedy and Eberhart (1995) as a stochastic optimization method. PSO is a population based search algorithm developed based on the simulation of the social behavior of bees, birds or a school of fish. Each individual within the swarm is represented by a vector in multidimensional search space. This vector has one assigned vector which determines the next movement of the particle called the velocity vector. The PSO also determines how to update the velocity of a particle. Each particle updates its velocity based on current velocity and the best position ( $\left.p \_b e s t\right)$ it has explored so far and the global best position ( $\left.g_{-} b e s t\right)$ explored by swarm. Movement of each particle is based on Eq. (6) and Eq. (7) depicted by Fig. 2. Eq. (6) shows that the velocity vector is updated by the global best position, personal best position, and current position of each particle. Eq. (7) shows that each particle moves by its velocity. 
$v_{i}(t+1)=a \cdot v_{i}(t)+b_{1} \cdot$ rand $\left(p_{-} \_\right.$best $\left.-x_{i}(t)\right)+b_{2} \cdot \operatorname{rand}\left(g_{-}\right.$best $\left.-x_{i}(t)\right)$,

$x_{i}(t+1)=x_{i}(t)+v_{i}(t)$

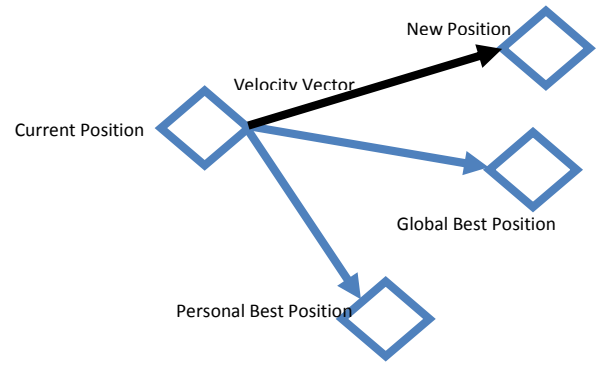

Fig. 2. Particle movement

where $i$ is the index of the particles, $t$ is the index of the iteration, $v_{i}$ is the vector of velocity, $x_{i}$ is the position, $a$ is the inertial weight, $b_{1}$ is the weight of difference between personal best and current position, $b_{2}$ is the weight of difference between global best and current position. Note that $a, b_{1}$ and $b_{2}$ are integer. Finally rand is used for randomization.

The pseudo code of the proposed PSO for APM is as follows:

1. Insert a decision matrix and weights of each criterion

2. Determine a, b1, b2, Max_Iter, and the number of particles, No_Particle, which is an integer number

3. Set $p$ Best_ $R=-\infty$ for personal best rate of each particle and gBest_ $R=-\infty$ for the global best rate, Generate random permutations for each particle and set Iter $=1$

4. Subtract a from the number of alternatives, for the number of the answer, exchange two alternatives randomly and repeat this step for each particle

5. Calculate the rate of each permutation $R$ using Eq. (4), for all particles. If $R>p B e s t \_R$ then $p B e s t=R$ and $p P e r=P e r$

6. Find the maximum rate and its permutation for this iteration, Entitle this rate and its permutation as $M R$ and MPer, respectively. If $M R>g B e s t \_R$, then $g B e s t \_R=M R$ and $g P e r=M P e r$

7. Generate a random integer number (RIN1) between 0 and b1. Liken RIN1 numbers of Per to pPer for each particle

8. Generate a random integer number (RIN2) between 0 and b2. Liken RIN2 numbers of Per to gPer for each particle

9. Iter=Iter+1, If Iter $<$ Max_Iter then go to step 4; otherwise the solution is gBest_R and gPer

The flowchart of this proposed procedure is depicted in Fig. 3. There are five parameters in the proposed PSO for APM. As mentioned before, the NOA is the important factor for tuning the parameters. Therefore, These parameters are set as: $N O_{-}$Particle $=15$, Max $\_$Iter $=5 \times N O A$, $a=N O A-1, \quad b_{1}=\lfloor N O A \div 2\rfloor$ and $b_{1}=\lfloor N O A \div 2\rfloor-2$. As we already explained we need to have $a \geq b_{1} \geq b_{2}$ for the implementation of our PSO and better results are normally expected using this condition (Clerc, 2006). This procedure can also flee from the local solution by using Eq. (6) and Eq. 
(7) with tuned parameters. The application and the implementation of the proposed meta-heuristics of APM are presented in the next section.

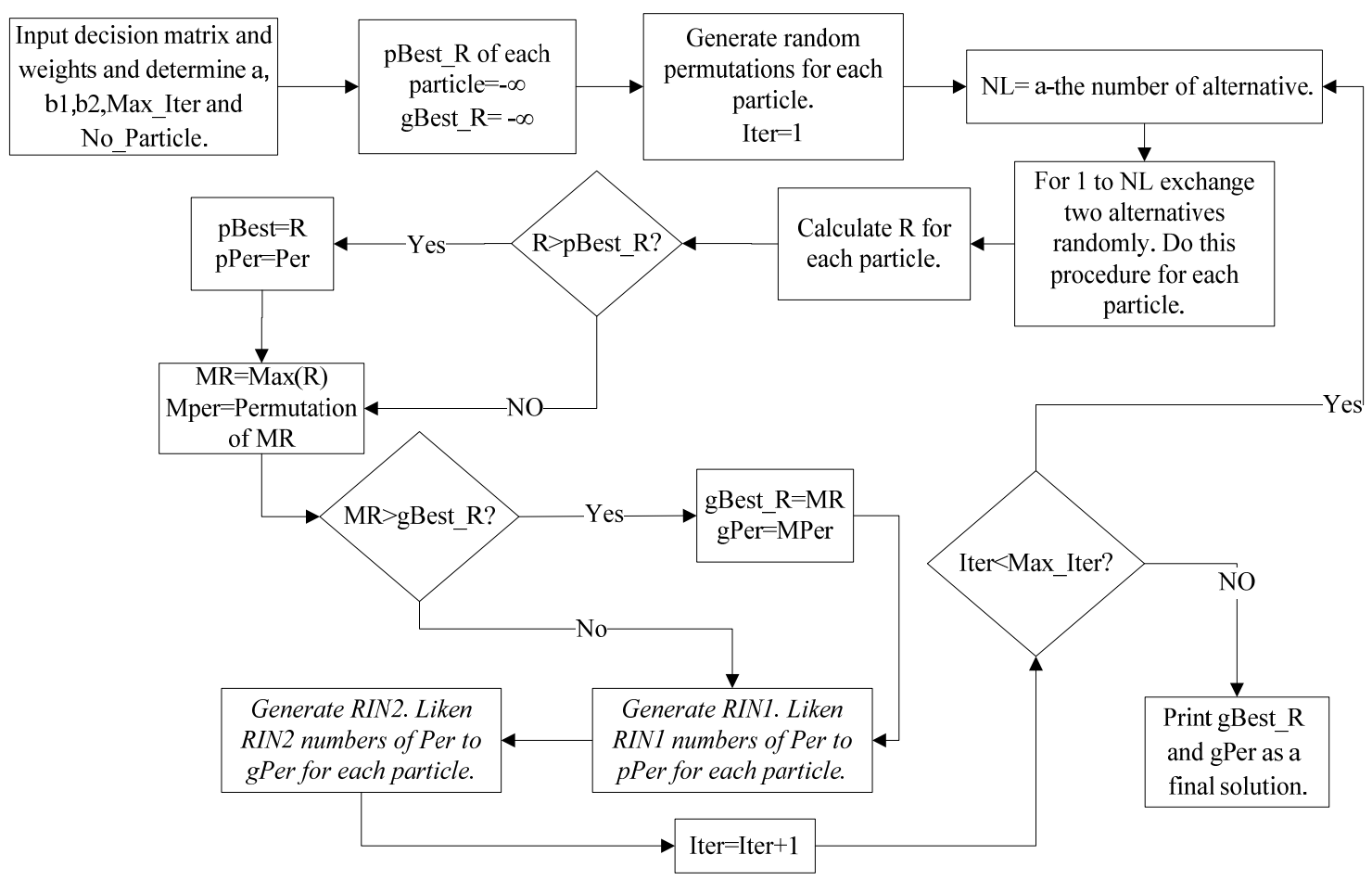

Fig. 3. Flowchart of the proposed PSO

\subsection{Illustrative examples}

In order to demonstrate the implementation of our proposed method we use two numerical examples in this section. The first example has 10 alternatives and 5 criteria where the second and the fifth criteria are negative attributes and the third and the fourth criteria are qualitative. The weights of the criteria are given as $0.3,0.2,0.1,0.1$ and 0.3 , respectively. Table 2 shows the decision matrix of this example.

\section{Table 2}

Decision matrix of the first example

\begin{tabular}{llllll}
\hline \multirow{2}{*}{ Alternative } & \multicolumn{5}{c}{ Criterion } \\
\cline { 2 - 6 } & $1(+)$ & $2(-)$ & $3(+)$ & $4(+)$ & $5(-)$ \\
\hline 1 & 22 & 4.2 & Very Good & Medium & 21 \\
2 & 25 & 2.6 & Good & High & 20 \\
3 & 24 & 1.5 & Poor & Low & 27 \\
4 & 15 & 6.8 & Good & Medium & 59 \\
5 & 35 & 7.9 & Medium & High & 67 \\
\hline 7 & 18 & 1.7 & Poor & Low & 34 \\
8 & 12 & 4.8 & Very Good & High & 26 \\
9 & 20 & 4.5 & Medium & Low & 34 \\
10 & 23 & 3.5 & Poor & Medium & 65 \\
\hline
\end{tabular}


To tackle this example, we first quantify the qualitative criteria. For the third criterion, "Poor", "Medium", "Good" and "Very Good" are transformed to 1, 2, 3 and 4, respectively. The fourth criterion is transformed in a similar manner. We determine the exact solution of this problem by checking all permutations and the example is solved using the two proposed meta-heuristics. The results are summarized in Table 3.

Table 3

Results of the first example

\begin{tabular}{lllllllllllll}
\hline $\begin{array}{l}\text { Solution } \\
\text { method }\end{array}$ & \multicolumn{10}{c}{ Rank } & \multicolumn{1}{c}{ Best rate $\begin{array}{l}\text { CPU } \\
\text { time (s) }\end{array}$} \\
\cline { 2 - 11 } & 1 & 2 & 3 & 4 & 5 & 6 & 7 & 8 & 9 & 10 & \\
\hline Exact & 2 & 1 & 7 & 5 & 3 & 8 & 6 & 10 & 9 & 4 & 9.1103 & 557.747 \\
TS & 2 & 1 & 7 & 5 & 3 & 8 & 6 & 10 & 9 & 4 & 9.1103 & 0.261 \\
PSO & 2 & 1 & 7 & 5 & 3 & 8 & 6 & 10 & 9 & 4 & 9.1103 & 0.987 \\
\hline
\end{tabular}

Table 3 shows that the proposed procedures replicate the solution of the exact method in a reasonable CPU time. In addition, it seems that the required CPU time for TS is less than PSO.

\section{Computational experiments}

This section presents the experiments conducted to investigate the performance of TS and PSO on benchmark instances which are generated randomly. We have generated a matrix with 30 alternatives and 6 criteria. These alternatives represent the suppliers of a corporation and the criteria are price, transportation cost, delivery time, history of cooperation, production capacity and quality, respectively. To generate these random instances, lower and upper bounds are considered for each criterion. These values for the first, the second and the fifth criteria are shown in Table 4 and the other criteria are qualitative. Hence, we use similar transformation method as explained before for quantitative values. Therefore, the values can be generated randomly and 5, 10, 15, 20 and 25 alternative subsets are built by taking the top $5 \times 6,10 \times 6,15 \times 6,20 \times 6$ and $25 \times 6$ submatrices and we can take 6 benchmark instances from this random problem.

Table 4

Lower and upper bounds for quantitative criteria in generating random matrix

\begin{tabular}{llll}
\hline & Lower bound & Upper bound & Type \\
\hline Price & 10 & 80 & Integer \\
Transportation Cost & 1 & 10 & Fraction \\
Production Capacity & 10 & 100 & Integer \\
\hline
\end{tabular}

In this matrix, the first and the second criteria are negative attributes. Weights of criteria are given as $0.3,0.2,0.2,0.05,0.2$ and 0.05 , respectively. The purpose of this problem is to find the preferable supplier and to rank these suppliers. Table 5 presents the random decision matrix of this benchmark problem. In line with the first example, the qualitative criteria are first quantified. In this example, the total number of permutations are 5!, 10!, 15!, 20!, 25! and 30! for the mentioned six benchmark instances, respectively. It is clear that tackling such large problem instances by complete enumeration 
of all permutations is impractical. This clearly calls for meta-heuristic procedures that can efficiently solve the problems.

We apply the TS and PSO procedures of subsection 4.1 and 4.2 to these instances and the results are repeated five different times. Table 6 and Table 7 summarize the details of our implementations. All computations were executed using MATLAB 7.8 on a personal computer equipped with 2.99GB of RAM and a Pentium microprocessor running at $2.53 \mathrm{GHz}$.

\section{Table 5}

Decision matrix of the benchmark problem

\begin{tabular}{|c|c|c|c|c|c|c|}
\hline \multirow[b]{2}{*}{ Alternative } & \multicolumn{4}{|c|}{ Criterion } & \multirow[b]{2}{*}{$5(+)$} & \multirow[b]{2}{*}{$6(+)$} \\
\hline & $1(-)$ & $2(-)$ & $3(+)$ & $4(+)$ & & \\
\hline 1 & 12 & 2 & Poor & Medium & 30 & Very Good \\
\hline 2 & 24 & 6 & Very Good & Low & 26 & Poor \\
\hline 3 & 35 & 1.5 & Poor & Low & 27 & Good \\
\hline 4 & 10 & 6.8 & Good & Low & 89 & Medium \\
\hline 5 & 45 & 9 & Poor & High & 59 & Very Good \\
\hline 6 & 58 & 7 & Poor & Low & 54 & Poor \\
\hline 7 & 12 & 8 & Very Good & Low & 16 & Medium \\
\hline 8 & 24 & 4.6 & Very Good & Medium & 67 & Poor \\
\hline 9 & 36 & 6.8 & Poor & Low & 80 & Good \\
\hline 10 & 20 & 3.2 & Medium & High & 46 & Poor \\
\hline 11 & 26 & 1.9 & Poor & High & 20 & Poor \\
\hline 12 & 69 & 8.9 & Very Good & Low & 26 & Poor \\
\hline 13 & 58 & 9 & Poor & Low & 38 & Poor \\
\hline 14 & 41 & 4 & Very Good & Low & 95 & Very Good \\
\hline 15 & 12 & 6 & Poor & High & 47 & Good \\
\hline 16 & 35 & 6.7 & Poor & High & 41 & Poor \\
\hline 17 & 30 & 6.9 & Medium & High & 16 & Very Good \\
\hline 18 & 20 & 4.6 & Good & Low & 57 & Very Good \\
\hline 19 & 70 & 5.8 & Poor & High & 50 & Poor \\
\hline 20 & 74 & 7 & Very Good & Medium & 90 & Poor \\
\hline 21 & 26 & 8.5 & Medium & Low & 13 & Very Good \\
\hline 22 & 70 & 3.6 & Good & High & 68 & Medium \\
\hline 23 & 53 & 9.4 & Medium & Medium & 53 & Good \\
\hline 24 & 36 & 2.2 & Poor & Low & 97 & Poor \\
\hline 25 & 16 & 1.4 & Very Good & Low & 36 & Very Good \\
\hline 26 & 41 & 5.1 & Good & High & 56 & Medium \\
\hline 27 & 28 & 9 & Medium & Medium & 78 & Poor \\
\hline 28 & 64 & 6.4 & Very Good & High & 83 & Good \\
\hline 29 & 31 & 4.2 & Poor & Low & 25 & Very Good \\
\hline 30 & 19 & 7 & Very Good & High & 47 & Poor \\
\hline
\end{tabular}


Table 6

The proposed TS results of the benchmark instances

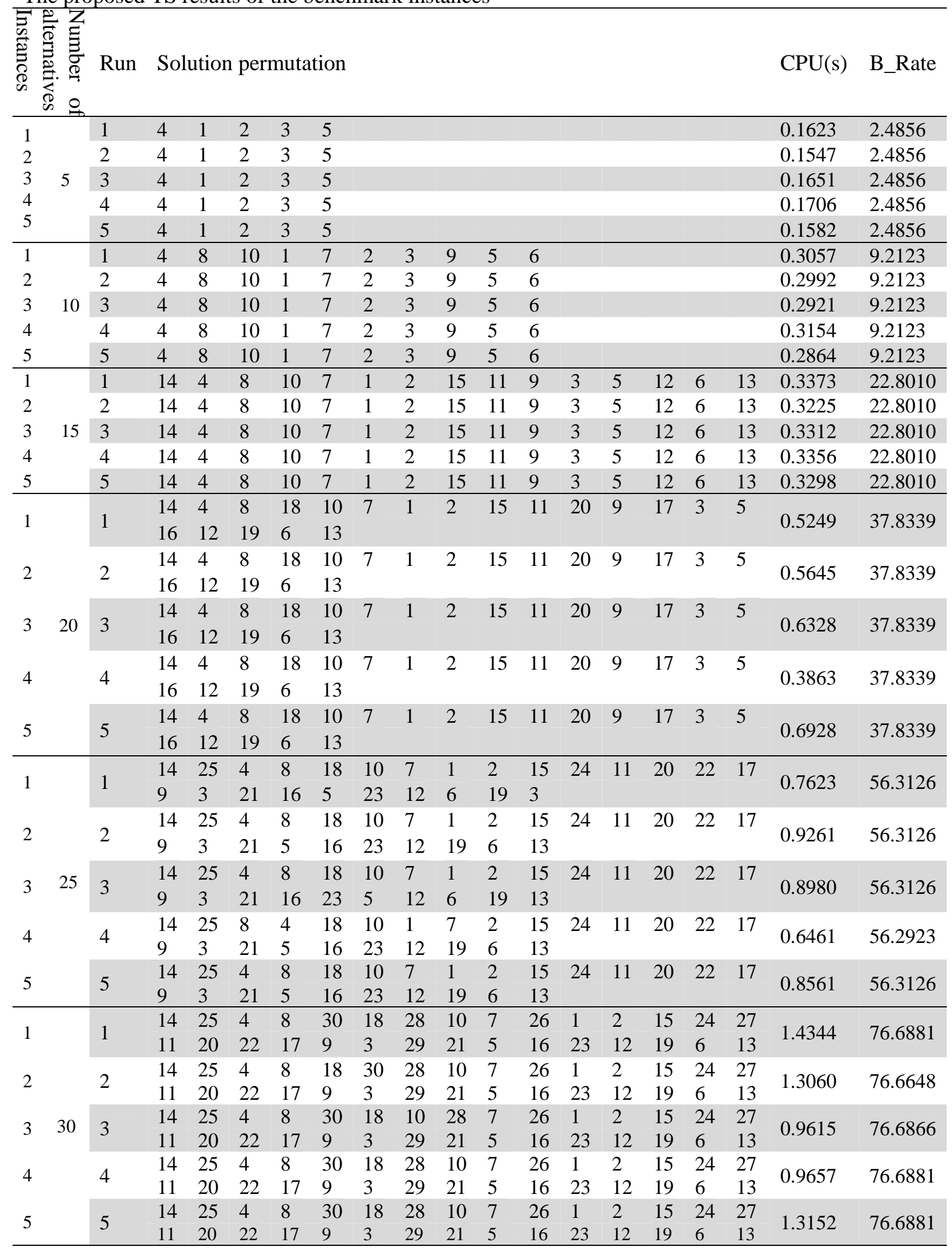


Table 7

The proposed PSO results of the benchmark instances

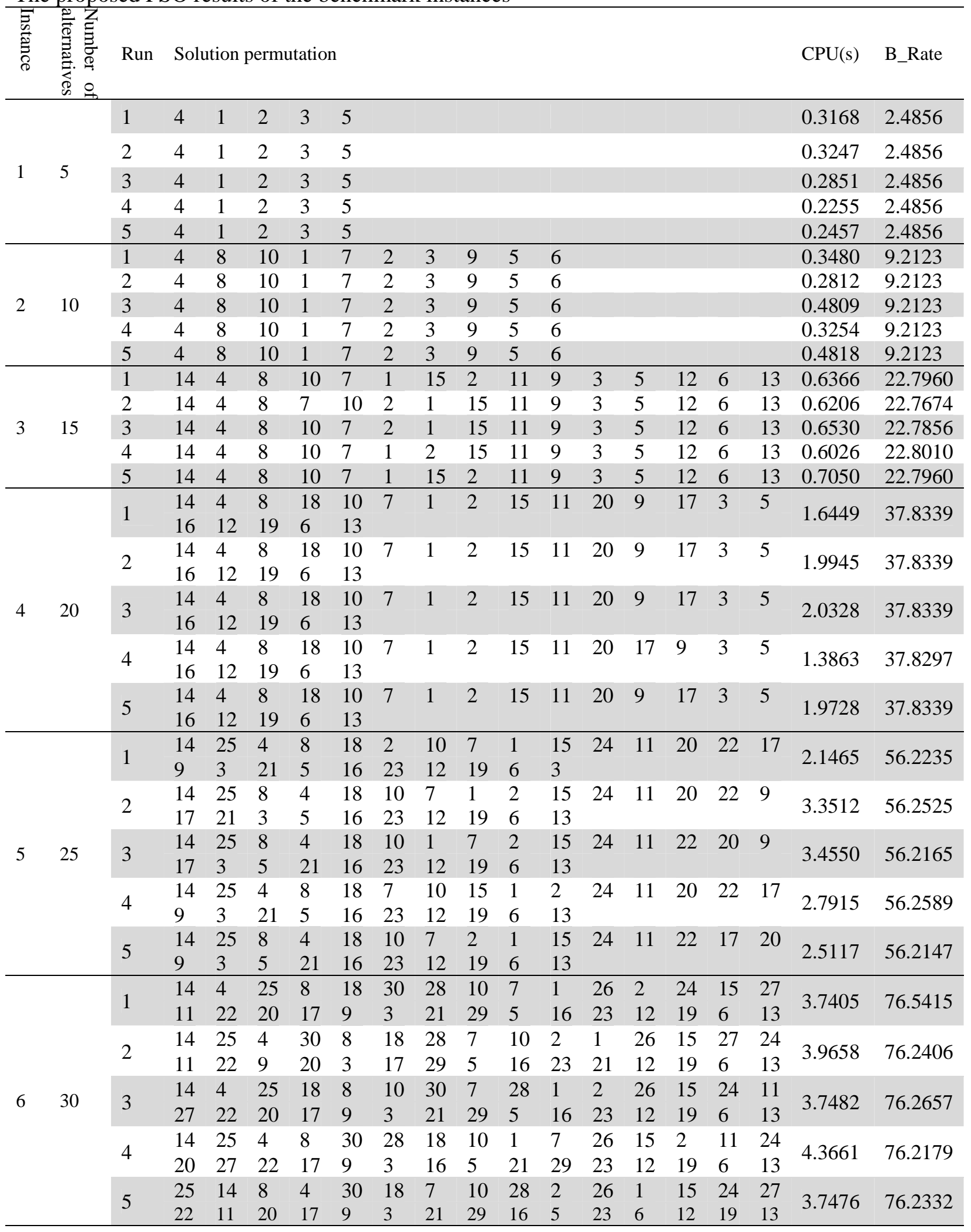




\subsection{Comparative Study}

In this section we study the performance of two proposed meta-heuristic TS and PSO approaches by choosing six benchmark instances and performing ANOVA test.
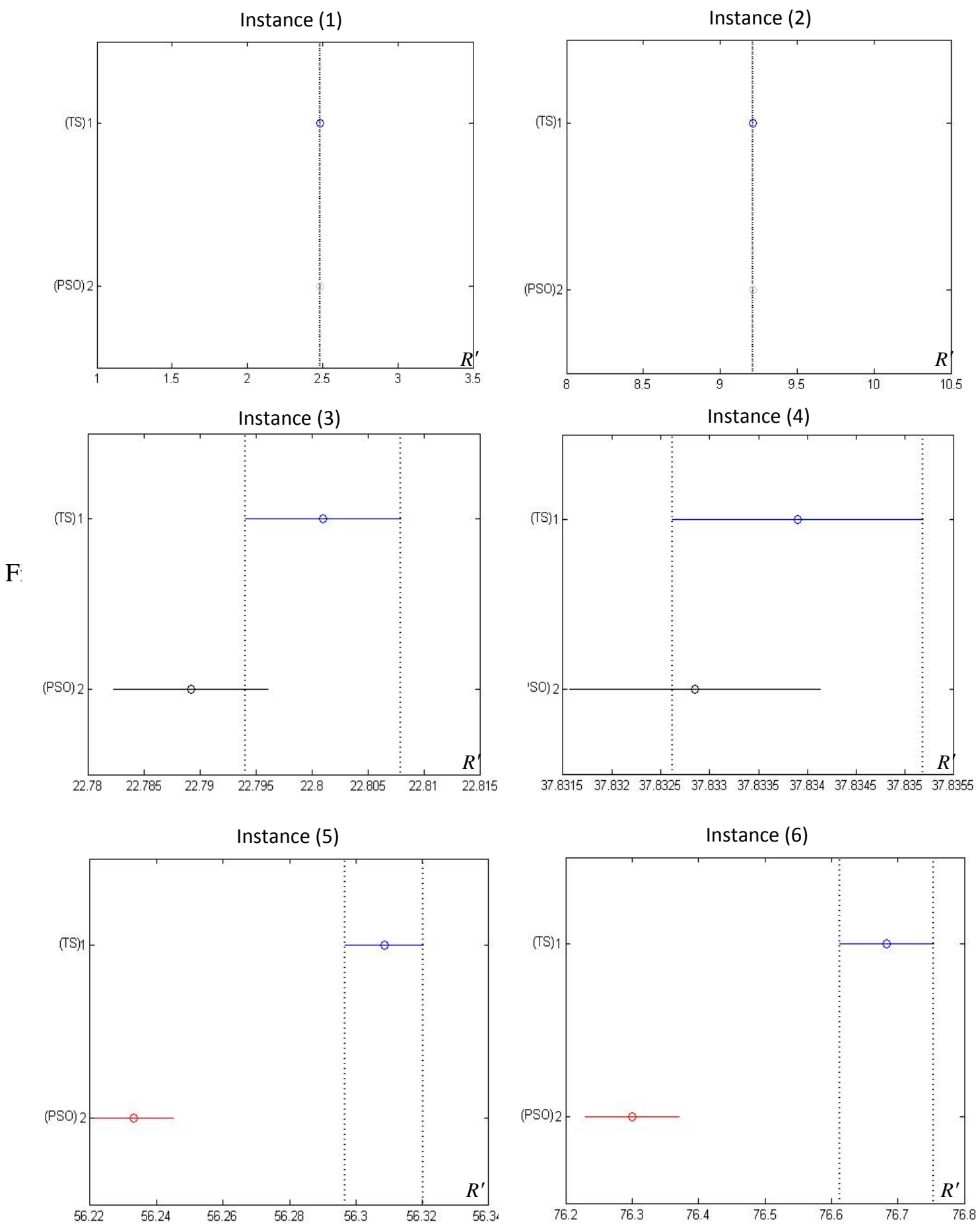

Fig. 4. ANOVA results: 95\% confidence interval for the difference between TS and PSO 
Fig. 4 summarizes the results of our investigation where the critical value for this analysis is considered as 0.05. As we can observe from Fig. 4 TS performs better compared with PSO method. Hence, it is not necessary that the $p$-values of these analyses to be declared.

In the first and the second instances which represent small instances, two meta-heuristic approaches have the same results. As we explained earlier, the performance of the proposed meta-heuristics cannot be measured based on the small instances. For the third and the forth instances which represent medum size instances we could observe some differences where TS seems to perform better than PSO.

The fifth and the sixth instances represent instances for large-scale problems. As we can see from the last two instances, the two approaches in APM perform completely different. Based on the results on Table 6 and Table 7 we can conclude that TS requires less CPU time than PSO for almost all cases. The results of these tables also show that the proposed TS in APM can nearly provide the exact solution.

One other observation from the experimental results is that both meta-heuristic approaches could solve the resulted NP-Hard permutation problem in reasonable amount time for typical real-world problems. The CPU times for most large-scale problems is less than a few seconds which means that a decision maker could solve a problem different times. This could be considered as an outstanding advantage since there are many cases where we may wish to run a problem under various cicumstances to perform sensitivity analysis.

\section{Conclusion}

In any MADM process, there are three important criteria which need to be considered. The first one is the selection of the significant criteria. The second one is the identification of the competent alternatives and last one is to make the decision based on an effective and efficient technique. The implementation of the third one normally involves the use of some NP-Hard approaches where we need to spend significant amount of time to reach optimal solutions.

We have presented a new adjustment permutation method for permutation scheme. The proposed method of this paper has been solved using two meta-heuristic approaches of TS and PSO. The preliminary results indicate that both meta-heuristic approaches could provide reasonable solutions but the proposed TS provides better results compared with PSO for large instances. As a future research direction, we propose applying other meta-heuristic procedures such as genetic algorithm and invasive weed optimization to the APM, and comparing results of this method with other MADM techniques.

\section{Acknowledgment}

The authors would like to thank the anonymous referees for their constructive comments on earlier version of this work. 


\section{References}

Ancot, J., \& Paelinck, J. H. P. (1982). Recent experiences with the Qualiflex multicriteria method. in: Paelinck, J. H. P. (Eds.), Qualitative and Quantitative Mathematical Economics., Martinus Nijhoff Publishers, pp. 217-266.

Blair, R., \& Karnisky, W. (1994). Distribution-free statistical analysis of surface and volumetric maps. Brain Topography, 6, 19-28.

Chen, T., \& Wang, J. (2009). Interval-valued fuzzy permutation method and experimental analysis on cardinal and ordinal evaluations. Journal of Computer and System Sciences, 75, 371-387.

Chin, L., \& Haughton, D. (1996). Analysis of student evaluation of teaching scores using bootstrap and permutation methods. journal of Computing in Higher Education, 8, 69-84.

Chu, T. (2002). Facility Location Selection Using Fuzzy TOPSIS under group decisions. International Journal of Uncertainty, Fuzziness and Knowledge-Based Systems, 10, 687-701.

Clerc, M.(2006). Particle Swarm Optimization. London: ISTE Ltd.

Figueira, J., Salvatore, G., Matthias, E. (2005). Multiple Criteria Decision Analysis: State of the Art Surveys. New York: Springer Science.

Glover, F. (1989). Tabu search: Part I. ORSA Journal on Computing, 1, 190-206.

Glover, F. (1990). Tabu search: Part II. ORSA Journal on Computing, 2, 4-32.

Grabowski, J., \& Pempera, J. (2007). The permutation flow shop problem with blocking. A tabu search approach. Omega, 35, 302-311

Grabowski, J., \& Wodecki, M. (2004). A very fast tabu search algorithm for the permutation flow shop problem with makespan criterion. Computers and Operations Research, 31, 1891-1909.

Hugonnard, J., \& Roy, B. (1982). Le plan d'extension du métro en banlieue parisienne, un cas type d'application de l'analyse multicritère. Les Cahiers Scientifiques de la Revue Transports, 6, 77108.

Jacquet-Lagreze, E. (1969). L’agrégation des opinions individuelles. en Informatiques et Sciences Humaines, 4, 1-21.

Kennedy, J., \& Eberhart, R. (1995). Particle swarm optimization. in: Proceedings of IEEE International Conference on Neural Networks, Piscataway, 1942-1948.

Korhonen, P., Moskowitz, H., \& Wallenius, J. (1992). Multiple Criteria Decision Support: A review. European Journal of Operational Research, 63, 361-375.

Liao, L., \& Huang, C. (2010). Tabu search for non-permutation flowshop scheduling problem with minimizing total tardiness. Applied Mathematics and Computation, 217(2), 557-567.

Nowicki, E., \& Smutnicki, C. (1996). A fast tabu search algorithm for the permutation flow-shop problem. European Journal of Operational Research, 91, 160-175.

Olson, D. L. (2004). Comparison of Weights in TOPSIS Models. Mathematical and Computer Modeling. 40(7-8), 721-727.

Peng, Y. (2000). Management Decision Analysis. Peking: Science Publications.

Paelinck, J. (1977). Qualitative multiple criteria analysis: an application to airport location. En'liironment and Planning, 9, 893-695.

Pantazis, D., Nichols, T., Baillet, S., \& Leahy, R. (2003). Spatiotemporal localization of significant activation in MEG using permutation tests. 18th Conference on Information Processing in Medical Imaging, 512- 523.

Rinnooy K. (1976). Machine Scheduling Problems: Classification, Complexity, and Computations, Nijhoff, The Hague.

Roy, B. (1968). Classement et choix en présence de critères multiples (la method ELECTRE), RIRO, 8, 57-75.

Roy, B., \& Bertier, B. (1971). Le methods ELECTRE II: Une methode de classement en presence de criteres multiples, note de travail no. 142. Direction Scientifique, Groupe Metra.

Roy, B., (1978). ELECTRE III: Un algorithme de classements fondé sur une représentation floue des préférences en présence de critères multiples. Cahiers du CERO, 20, 3-24. 
Saaty, T. L. (1990). The Analytic Hierarchy Process, McGraw-Hill, RWS Publications, Pittsburgh, PA.

Tasgetiren, M. F., Liang, Y. C., Sevkli, M. \& Gencyilmaz, G. (2007). A particle swarm optimization algorithm for makespan and total flowtime minimization in the permutation flowshop sequencing problem. European Journal of Operational Research,177, 1930-1947.

Turskis, Z. (2008). Multi-Attribute Contractors Ranking Method by Applying Ordering of Feasible Alternatives of Solutions in Terms of Preferability Technique. Technologic and Economic Developement, 14, 224-239.

Yoon, K., \& Hwang, C. (1981). Multiple Attribute Decision Making Method and Applications. Berlin: Springer. 